\title{
Review Article \\ Cryopreservation of Mammalian Oocyte for Conservation of Animal Genetics
}

\author{
Jennifer R. Prentice ${ }^{1,2}$ and Muhammad Anzar ${ }^{1,2}$ \\ ${ }^{1}$ Canadian Animal Genetic Resources, Agriculture and Agri-Food Canada, Saskatoon Research Centre, \\ 107 Science Place, Saskatoon, SK, Canada S7N 0X2 \\ ${ }^{2}$ Department of Veterinary Biomedical Sciences, Western College of Veterinary Medicine, University of Saskatchewan, \\ 52 Campus Drive, Saskatoon, SK, Canada S7N 5B4
}

Correspondence should be addressed to Muhammad Anzar, muhammad.anzar@agr.gc.ca

Received 3 June 2010; Accepted 9 August 2010

Academic Editor: Stefan Schlatt

Copyright (C) 2011 J. R. Prentice and M. Anzar. This is an open access article distributed under the Creative Commons Attribution License, which permits unrestricted use, distribution, and reproduction in any medium, provided the original work is properly cited.

\begin{abstract}
The preservation of the female portion of livestock genetics has become an international priority; however, in situ conservation strategies are extremely expensive. Therefore, efforts are increasingly focusing on the development of a reliable cryopreservation method for oocytes, in order to establish ova banks. Slow freezing, a common method for cryopreservation of oocytes, causes osmotic shock (solution effect) and intracellular ice crystallization leading to cell damage. Vitrification is an alternative method for cryopreservation in which cells are exposed to a higher concentration of cryoprotectants and frozen with an ultra rapid freezing velocity, resulting in an ice crystal free, solid glass-like structure. Presently, vitrification is a popular method for cryopreservation of embryos. However, vitrification of oocytes is still challenging due to their complex structure and sensitivity to chilling.
\end{abstract}

\section{Introduction}

Many domestic breeds of livestock are experiencing a gradual diminishment of genetic diversity; therefore, it is in the interest of the international community to conserve the livestock genetics. Ideally populations are saved as live animals; however, this approach is expensive, and unless the breed can be used for production, it is not likely to succeed. Therefore, ex situ in vitro conservation strategies are developed to cryopreserve animal genetic resources in genome/gene banks to regenerate a particular population in future $[1,2]$. Although significant progress has been made in both semen and embryo cryopreservation of several domestic species, oocytes are extremely sensitive to chilling, and to date a standardized procedure has not been established. Long-term storage of oocytes would develop of ova banks, permitting female genetic material to be stored unfertilized until an appropriate male germplasm is selected. Successful cryopreservation of oocytes would also preserve the genetic material from unexpectedly dead animals and facilitate many assisted reproductive technologies [3-5].

\section{Loss of Farm Animal Genetic Diversity}

In the last few decades, farm animal genetic diversity has rapidly declined, mainly due to changing market demands and intensification of agriculture. Agriculture is moving away from small production systems to large commercial systems, and as a result, selection goals and production environments are now very similar throughout the world. Modern reproductive technologies have allowed a large number of progeny to be produced from a single individual,and contemporary transport has enabled the distribution of germplasm around the world rapidly and efficiently. Livestock diversity has also been diminished by many breeding programs carried out by national and international companies, which place intense selection pressure on few breeds [6].

According to the FAO, approximately 20 percent of the world's breeds of cattle, goats, pigs, horses, and poultry are currently at risk of extinction, and at least one livestock breed has become extinct per month over the past several years, resulting in its genetic characteristics being 
lost forever [7]. It is imperative to conserve and maintain animal genetic resources to ensure the ability to respond to selection plateaus, consumer demand changes but more importantly biosecurity, environmental, and food safety risks by maintaining biodiversity and keeping alternative and potentially useful genes available in the gene pool $[3,8]$.

\section{Strategies for Farm Animal Genetic Conservation}

Increasing awareness on the reduction of breed diversity has prompted global efforts for conservation of threatened breeds through launching organizations such as Rare Breeds Canada, conferences like the Convention on Biological Diversity, and programs such as the Canadian Animal Genetic Resources. Efforts have focused on conservation of farm animal breeds for several reasons, for example, to keep potentially useful genes and gene combinations, to take advantage of heterosis, and to overcome selection plateaus, as well as for cultural reasons, research, and food security. Maintaining genetic diversity also provides insurance against climate change, disease, changing availability of feedstuffs, social change, selection errors, and unforeseen catastrophic events such as Chernobyl where many local breeds' diversity became threatened $[6,9,10]$.

The goals of conservation are to keep genetic variation as gene combinations in a reversible form and to keep specific genes of interest such as the Booroola fecundity gene in sheep [11]. In order to achieve these goals several steps are necessary. First an inventory must be taken to assess and monitor the risk status of a breed in an ongoing basis by examining the number of breeding males and females, the overall breed numbers, the number of subpopulations, and the trends in population size. The evaluation of stocks for phenotype and genotype must be done to determine the genetic distance of one group from another and the choice of breeds for conservation [6].

3.1. In Situ Conservation. Ideally, populations should be saved as live animals through in situ conservation programs; however this approach needs extensive infrastructure and management and thus is expensive [6]. Hence, ex situ in vitro strategies have been developed to cryopreserve animal genetic resources in a genome bank that creates a global gene pool to manage the exchange of genetic diversity or regenerate a population decades or centuries later $[1,2]$. The Convention on Biological Diversity recommended that ex situ conservation be complementary to in situ conservation for farm animal genetic resources [12].

3.2. Ex Situ Conservation. One of the major issues surrounding genome banks is the amount and type of material that needs to be stored, which is a function of the intended future use of the material [12]. In order to avoid inbreeding, a gene bank of male and female genetics formed from the largest number of individuals would be ideal [13]. Ex situ in vitro conservation programs of livestock genetic resources have focused efforts on cryopreservation of gametes, embryos, and somatic cells as well as testis and ovarian tissues, effectively lengthening the genetic lifespan of individuals in a breeding program even after the death $[1,8]$.

3.2.1. Semen. Semen is one of the most practical means of storing germplasm due to its abundant availability and ease of application $[1,14]$. Stored spermatozoa could be introduced back into existing populations either immediately or decades or centuries afterwards. Stored frozenthawed semen from genetically superior males of threatened livestock breeds could be used for artificial insemination (AI) or in vitro fertilization (IVF) and has the potential to protect existing diversity and maintain heterozygosity while minimizing the movement of living animals $[8$, 15]. Breed reconstruction solely from semen is possible through a series of back-cross generations; however, the entire genetics of the original breed will not be recovered [12].

Semen from most mammalian and a few avian species has been successfully frozen in the past several years [14]. However, the protocols currently used to conserve semen are still suboptimal and cannot be easily applied across species [16]. First-service conception rates vary drastically between different breeding programs, but on average conception rates are fairly high in cattle, pigs, goats, and sheep.

3.2.2. Embryos. Embryo cryopreservation allows the conservation of the full genetic complement of both dam and sire and has tremendous opportunities for maintaining heterozygosity and population integrity, but it is more complex and costly procedure than semen cryopreservation. Moreover, a large number of embryos would be required for complete reconstruction of a population and are unlikely to be available from donor females of endangered breeds [12]. Embryos of virtually all mammals have been successfully frozen, thawed, and transferred to synchronized recipient females in the past; however, embryos from species such as swine or equine are much more cryosensitive compared to bovine or ovine embryos. Currently, the widespread use of embryo cryopreservation is limited to cattle, sheep, and goats $[17,18]$. Table 1 demonstrates embryo sensitivity towards cryopreservation in different species, developmental stages, and origin. It is apparent that earlier and in vivo derived embryos withstand cryopreservation better than later stage and in vitro produced embryos. Therefore, the current challenge is to develop a standardized protocol that can be applied to embryos of different species at various developmental stages $[3,14,19]$.

3.2.3. Oocytes. Oocytes are large cells, with a low surface to volume ratio, surrounded by zona pellucida. Immediately adjacent to the oocyte are corona radiata cells that have long cytoplasmic extensions which penetrate the zona pellucida, ending in oocyte membrane. These processes and gap junctions are important in the metabolic cooperation between the oocyte and surrounding layers of granulosa cells, which form the cumulus-oocyte complex (COC) during the growth phase. 
TABLE 1: Differences of animal oocyte and embryo cryopreservation resistance among species, developmental stages, and origin.

\begin{tabular}{lcc}
\hline & More resistance & Less resistance \\
\hline Species & Bovine, ovine & Porcine, equine \\
$\begin{array}{l}\text { Developmental } \\
\text { Stages }\end{array}$ & $\begin{array}{c}\text { Morula, YBL, and } \\
\text { BL }\end{array}$ & Hatched BL and oocytes \\
Origin & $\begin{array}{c}\text { In vivo derived } \\
\text { embryos }\end{array}$ & $\begin{array}{c}\text { In vitro produced embryos, } \\
\text { micromanipulated embryos }\end{array}$ \\
\hline
\end{tabular}

YBL: young blastocyst; BL: blastocyst. Adapted from Pereira and Marques, 2008 [3].

Oocytes collected from slaughterhouse derived ovaries are at the germinal vesicle (GV) stage in which the genetic material is contained within the nucleus. Since this stage has no spindle present, GVs are assumed to be less prone to chromosomal and microtubular damage during cryopreservation. However, oocytes can also be cryopreserved at the metaphase II (MII) stage of maturation. During MII stage, the cumulus cells surrounding the oocyte are expanded, microfilaments of actin are involved in cell shape and movements, and microtubules form the spindle apparatus [20].

Oocytes collected by in vivo pickup or at slaughter can be frozen for extended periods of time for subsequent IVF to produce embryos. Oocyte banks would enlarge the gene pool, facilitate several assisted reproductive procedures, salvage female genetics after unexpected death, and avoid controversy surrounding the preservation of embryos [4, 5]. Like semen, oocyte cryopreservation is beneficial for international exchange of germplasm, as it avoids injury and sanitary risks involved in live animal transportation [3].

Oocytes are extremely sensitive to chilling, and the technique is not as established as in semen or embryos, due to the fact that oocytes typically have a low permeability to cryoprotectants [16]. The major differences between oocytes and embryos are the plasma membrane, presence of cortical granules, and spindle formation at metaphase II (MII) stage of meiosis [21]. To date, there has been no consistent oocyte cryopreservation method established in any species, although, there has been significant progress and offspring have been born from frozen-thawed oocytes in cattle, sheep, and horses [16, 22, 23]. During the process of cryopreservation, oocytes suffer considerable morphological and functional damage, although, the extent of cryoinjuries depends on the species and the origin (in vivo or in vitro produced). The mechanism for cryoinjuries is yet to be fully understood, and until more insight is gained, improvement of oocyte cryopreservation will be difficult [3].

\section{Oocyte Cryopreservation}

4.1. Principles. Cryopreservation involves cells or whole tissues preservation by exposure to subzero temperature in $\mathrm{LN}_{2}\left(-196^{\circ} \mathrm{C}\right)$ [16]. At such a low temperature, biological activity is effectively stopped, and the cells functional status may be preserved for centuries [24]. However, several physical stresses damage the cells at these low temperatures. Intracellular ice formation is one the largest contributors to cell death; therefore, freezing protocols use a combination of dehydration, freezing point depression, supercooling, and intracellular vitrification in an attempt to avoid cell damage [25].

It is important to consider the nature and concentration of the cryoprotectant(s) for preservation of germplasm in any cryopreservation protocol. Dimethylsulfoxide (DMSO), ethylene glycol (EG), or glycerol alone or in combination protects the cells and tissues from freezing damage. Moreover, the cooling rate and freezing method are also important factors to consider in preventing cryoinjuries of cells. Slow freezing, using a controlled freezing curve, is commonly used for cell cryopreservation; however, vitrification, in which high concentrations of cryoprotectants and ultra rapid freezing velocity are used, is gaining popularity due to its promising success rates in certain species [3].

4.2. Cryoinjuries. During cryopreservation, the extent of injury incurred in cells largely depends on the size and shape of the cell, the permeability of the membranes, and the quality of the oocyte. However, these factors vary between species, developmental stage, and origin [26]. Although offspring have been born using frozen-thawed oocytes from various species, the ability to support embryo development following cryopreservation procedures is low. This may be attributed to the susceptibility of oocytes to damage during cooling and/or freezing and subsequent thawing because of their complex structure. Unfertilized mammalian oocytes are much larger therefore have small surface:volume ratio [21]. This makes dehydration and penetration of cryoprotectants difficult to achieve, which contributes to the complexity in cryopreservation. Moreover, the plasma membrane of oocyte differs drastically from that in embryo. Following fertilization, there is a rise in intracellular free calcium, which modifies the ionic strength and membrane potential of the plasma membrane [27]. The submembranous polymerized filamentous actin concentration increases, and its conformation changes, which facilitate the permeation of water and cryoprotectants that promotes dehydration and reduces intracellular ice crystal formation. Additionally, the higher strength of the cell membrane increases the osmotic tolerance during thawing and allows embryos to withstand freezing and thawing better than oocytes [21].

Freezing immature, in vitro matured or ovulated oocytes often results in morphological and functional damage. Although the specific molecular pathways disrupted during freezing are not well understood, many ultrastructural elements that are critical to maintenance and development are damaged [28]. Postthaw oocytes often exhibit zona pellucida or cytoplasmic membrane fractures, and cooling oocytes from approximately $37^{\circ} \mathrm{C}$ to $20^{\circ} \mathrm{C}$ or below can result in various cytoskeletal and chromosomal modifications, although some oocytes have the ability to fully or partially repair themselves $[20,26]$. The major adverse consequences following freezing procedures are due to ice crystal formation, osmotic injury, toxic effects of cryoprotectants, 
concentrated intracellular electrolytes, and chilling which lead to zona fracture, alterations in intracellular organelles and cytoskeleton $[29,30]$. Strategies to overcome these manifestations involve reducing container volumes, increasing the thermal gradient, altering the cell surface to volume ratio, and the addition of substances to increase cryotolerance [3]. The addition of molecules such as antifreeze proteins, sugars, or antioxidants have been shown to stabilize the membrane during cooling [4].

Various meiotic stages exhibit different sensitivities to freezing. Oocytes may be cryopreserved at immature GV stage or at mature MII stage. Reports have shown that freezing immature oocytes is ideal as there is no meiotic spindle present and the genetic material is confined within the nucleus; however, immature oocytes are thought to be more sensitive to anastomotic stress and have lower cell membrane stability than MII stage oocytes [4, 31-33]. The success of immature oocyte cryopreservation largely depends on the ability to preserve the structural and functional integrity of the entire oocyte and cumulus cells surrounding the oocyte. The gap junctions between oocytes and cumulus cells play an important role in the maturation process by providing nutritive substances that have a supportive role during IVF [20]. It has been demonstrated that GV stage oocytes stripped of cumulus cells exhibit deficient nuclear and cytoplasmic maturation [31, 34, 35]. However, cumulus cells can also be an obstacle to the penetration of cryoprotectants [30].

Cooling immature oocytes below $4^{\circ} \mathrm{C}$ hinders the formation of meiotic spindles and fertilization while exposing mature oocytes to cryoprotectants, and low temperatures can result in damage to the meiotic spindle, actin filaments, and chromosomal dispersal and microtubule depolymerization $[20,36]$. Abnormalities in the meiotic spindle is related with the loss of fertilization and embryo development as spindle is crucial for completion of meiosis, second polar body formation, migration of the pronuclei, and formation of the first mitotic spindle [37]. Spindle disorganization can result in chromosomal dispersion, failure of normal fertilization, and incomplete development $[21,38]$.

The microtubule, which is a component of the spindle, is a cylindrical bundle, comprising 13 protofilaments, a heterodimer consisting of $\alpha$ and $\beta$-tubulin. Microtubules begin from microtubular organizing centers at both poles and anchor chromosomes at the kinetochores [21]. The chromosomes align at the equatorial plane of the meiotic spindles. A recent study in porcine oocytes found that paclitaxel treatment improved the normality of microtubules by strengthening the bond between $\alpha$ and $\beta$-tubulin and improved the developmental ability of vitrified MII oocytes $[36,39]$.

Regardless the stage of oocytes, DNA is damaged during cryopreservation [20]. Cryopreserved oocytes have altered distribution of cortical granules, increased polyspermy, and zona hardening due to premature cortical granule release, which deters the entry of sperm and thus fertilization $[3,39-$ 42]. The use of intracytoplasmic sperm injection (ICSI) overcomes the effects of zona hardening. In 1995, using ICSI for frozen-thawed oocytes resulted in higher fertilization
TABLE 2: Comparison of oocyte and embryo cryopreservation methods.

Freezing procedures
$\begin{aligned} & \text { Conventional slow-freezing } \\ & \text { method }\end{aligned}$

method

(1) Several devices for loading embryos and oocytes

(1) Standard $0.25 \mathrm{ml}$ straws

(conventional straws, open pulled straw, cryoloop, cryoleaf, cryotop, etc.)

(2) High cryoprotectant

(2) Low cryoprotectant concentration/ reduced volume concentration and time with vitrification solution

(3) Seeding at -5 to $-7^{\circ} \mathrm{C}$, (3) Ultra-rapid cooling rates controlled slow cooling $\left(0.1 \quad\left(-2500^{\circ} \mathrm{C} / \mathrm{min}\right.\right.$ or $20000^{\circ} \mathrm{C} / \mathrm{min}$ to $0.3^{\circ} \mathrm{C} / \mathrm{min}$ ) using OPS and cryoloop)

(4) Plunging at -30 to

$-70^{\circ} \mathrm{C}$ and storage in

liquid nitrogen $\left(-196^{\circ} \mathrm{C}\right)$

(4) Plunging into liquid nitrogen $\left(-196^{\circ} \mathrm{C}\right)$

Adapted from Pereira and Marques, 2008 [3].

rates than IVF; however, in animals the use of ICSI is not as established as IVF and thus requires further investigation $[21,43]$.

4.3. Freezing Procedures. Currently, two main methods for cryopreservation of oocytes are slow freezing and vitrification. With the exception of cryoprotectants concentration and cooling rate, these two methods differ slightly with regards to storage, warming, and rehydration [26]. Table 2 compares conventional freezing and vitrification methods for oocyte and embryo cryopreservation [3].

Conventional slow freezing was introduced first and is currently the gold standard for cryopreservation of embryos. This method typically involves the use of a single cryoprotectant in low concentrations (approximately 1 to $2 \mathrm{M}$ ) to minimize chemical and osmotic toxicity and attempts to maintain a balance between the various factors that influence cell damage [3]. During the controlled cooling rate, water is exchanged between the extracellular and intracellular fluids without serious osmotic effects [26]. However, during slow cooling, extracellular water precipitates as ice resulting in ice crystal formation. Slow freezing gives acceptable results for oocytes of species that are not sensitive to chilling such as cat [44], human [45], and mouse [46]. However, bovine and porcine oocytes are more sensitive to chilling and yield poor results following slow cooling [4].

The physical definition of vitrification is the glass-like solidification of solutions at low temperatures, without the formation of intracellular ice crystals. During this method of cryopreservation, ice crystal formation is prevented due to the viscosity of the high concentrations of cryoprotectants (approximately 7 to $8 \mathrm{M}$ ) used in vitrification media that makes water solidify without expansion [30]. Cells undergoing vitrification are frozen at an extremely rapid cooling rate and often undergo fewer physiological detrimental effects 
compared with slow freezing [47]. Although, some transitional and very short freezing of the solutions can occur during warming following vitrification, this is generally harmless to the oocyte. However, the high concentrations make cryoprotectants toxic to the cells. As a result, oocytes can only be exposed to a minimal volume of vitrification media for a very short time, that is, $<1 \mathrm{~min}$ [3]. Extensive research in the past 20 years has resulted in new approaches which have created an acceptable balance between the positive and negative effects of vitrification. Ultra-rapid cooling rates have allowed decreased cryoprotectant concentrations and have made vitrification an extremely competitive alternative to conventional slow freezing [26]. Presently, vitrification is a popular method for cryopreservation of many different cell types, tissues, and organs; however, the extent of cryoinjury and developmental rates are highly variable depending on the species $[3,20]$.

Despite the fact that slow freezing is the most widely used cryopreservation technique, vitrification is a viable and promising alternative that is increasingly becoming more attractive to the commercial sector. Many reports comparing conventional embryo slow freezing and vitrification have reported either equal or better in vitro or in vivo survival rates following vitrification $[48,49]$. Vitrification of oocytes and embryos has been tested in several species with good results, does not require costly coolers or special skill, and can be performed fairly quickly [30, 50-52]. It has been suggested that with time, conventional slow freezing will be replaced entirely by vitrification techniques [26].

4.4. Cryoprotectants. Cryopreservation strategies are based on two main principles: cryoprotectants and coolingwarming rates [26]. Because water is not very viscous, it can only be vitrified by extremely rapid cooling of a small sample or using high concentrations of cryoprotectants [53]. Cryoprotectants such as glycerol, dimethyl sulfoxide (DMSO), and ethylene glycol (EG) are small molecules that penetrate cells and limit the amount of intracellular and extracellular water that converts into ice during cooling. However, cells do have a biological limit to tolerate the concentration of cryoprotectants. It is imperative to maximize the cooling rate while minimizing the concentration of cryoprotectants during vitrification [53].

Cryoprotective solutions are typically prepared in buffered media (e.g., TCM-199) with a stable $\mathrm{pH}$ between 7.2 and 7.4 [54]. Cryoprotectants are compounds used in cryoprotective solutions to achieve cellular dehydration and to avoid intracellular ice crystal formation upon freezing. Typically combinations of cell permeating and nonpermeating cryoprotectants are used. Low molecular weight permeating cryoprotectants such as glycerol, EG, and DMSO are small molecules that enter the cell, form hydrogen bonds with intracellular water molecules, and lower the freezing temperature, preventing crystallization. Low molecular weight non-permeating cryoprotectants such as sucrose, glucose, trehalose, and fructose remain extracellular and draw free water out of the cell by osmosis, resulting in intracellular dehydration [3]. The addition of sugars to an EG-based media can strongly influence the vitrification properties of the solution and assist in stabilizing membrane structures [55]. Disaccharides act as osmotic buffers to reduce osmotic shock and the toxicity of EG by decreasing the concentration required to achieve successful cryopreservation [53].

High molecular weight non-permeating polymers or macromolecules are commonly used to reduce the amount of intracellular cryoprotectants necessary for vitrification, reducing the toxicity of the solution. Polymers protect zona pellucida against cracking. The majority of solutions used for oocyte vitrification contain a macromolecular component of fetal calf serum or bovine serum albumin. Other macromolecules used in vitrification media include polyethylene glycol, polyvinylpyrrolidone, Ficoll, and polyvinyl alcohol [3].

The addition of nonpermeating polymers, a combination of more than one cryoprotectant, and their stepwise exposure minimizes the toxic effect of cryoprotectants on cells. Additionally, it has been shown that adding cells to a lower concentration of EG before transferring them to a higher concentration disaccharide mixture can reduce the toxic effect of cryoprotectants [53].

4.5. Cooling Rate. It has been reported that a cooling rate of approximately $2500^{\circ} \mathrm{C} / \mathrm{min}$ can achieve a vitrified state [54]. However, using a suitable carrier system such as the open pulled straw, a cooling rate of $20000^{\circ} \mathrm{C} / \mathrm{min}$ can be achieved [50]. The main reasons for increasing cooling and warming rates are to avoid chilling injury and to decrease the concentration of cryoprotectants in solution [53]. By passing cells through the critical temperature zone (15 to $-5^{\circ} \mathrm{C}$ ) quickly, water moves out of the cells and freezes extracellularly [20]. This prevents chilling injury to the intracellular lipid droplets, lipid containing membranes, and the cytoskeleton [30].

As cells are immersed in $\mathrm{LN}_{2}$, it is warmed, resulting in extensive boiling. Evaporation occurs, and a vapor coat surrounds the cells and creates an insulative layer that decreases the temperature transfer and cooling rate. Minimizing the volume surrounding the cell, avoiding $\mathrm{LN}_{2}$ vapor formation, and establishing direct contact between the cryoprotectant and the $\mathrm{LN}_{2}$ all assist in increasing the cooling and warming rates during vitrification of cells [53]. However, techniques based on the direct contact of $\mathrm{LN}_{2}$ and the medium containing the oocytes may be a source of contamination. These risks can be minimized by using sterile $\mathrm{LN}_{2}$ for cooling then wrapping the oocytes in a hermetic container before storage; however, this procedure may be too complex for everyday application [30].

The development of special carriers such as the openpulled straw [56], microdrops [57], cryoloop [58], flexipetdenuding pipette [59], electron microscopic copper grids [60], hemistraw system [61], small nylon coils [62], nylon mesh [63], and cryotops [64] has achieved higher cooling rates while permitting the use of less toxic and less concentrated solutions [20]. These carriers shorten the time of exposure with the final cryoprotectant before cooling and after warming, and the small volume of solution prevents heterogeneous ice formation [30]. Consequently, 
the promising results have led to new method to increase the cooling rate using supercooled $\mathrm{LN}_{2}[53,65]$.

In order to avoid cell injury and death during vitrification, ice crystal formation needs to be prevented by removing as much of the intracellular water as possible. However, the removal of excessive water results in cell injury and death through the effect of the highly concentrated intracellular environment on membranes, referred as "solution effect" [53].

4.6. Criteria to Assess the Quality of Frozen-Thawed Oocytes. There are several different methods used to test the viability and extent of chilling injuries of oocytes following cryopreservation. Typically, the primary criteria used to assess postthaw viability of oocytes are the presence or absence of degeneration or cytoplasmic vascularisation or zona pellucida fractures [66]. The membrane damage was evaluated using probes to indicate the integrity of the plasma membrane [4]. Recent studies examined the meiotic spindle in human oocytes, using a polarized microscope apparatus, which allows the visualization of the polymerisation of the meiotic spindle following warming. However, this technique is difficult in domestic animals due to their high cytoplasmic lipid content, which hinders spindle examination. Therefore, the oocytes of domestic animals are typically examined through invasive methods such as fluorescence microscopy and biochemical or molecular analyses [66].

Current research is focusing on the development of new noninvasive evaluation techniques or markers. For example, if the gene expression of the cumulus cells could be correlated with developmental rates, gene expression could be used as a marker for oocyte quality before freezing, helping to select the oocytes most suitable for cryopreservation [67]. Moreover, determining the volumetric response of matured oocytes to changes in osmolarity during preparation for cooling would be another non-invasive response to oocyte evaluation. This may assist in decreasing the toxic and osmotic effects of cryoprotectants on oocytes. Measuring the volumetric response to increasing cryoprotectants concentrations permits a precise estimate of the ideal timing and concentration of cryoprotectants exposure [66].

Although the viability of frozen-thawed oocytes has been tested using in vitro fertilization and ICSI, the best way to evaluate the capacity of embryos derived from cryopreserved oocytes is to produce viable offspring [68]. Currently there have been live offspring born from cryopreserved oocytes in humans [69] and bovine [70].

\section{Vitrification of Oocytes}

Over the years there has been considerable effort focused on reducing the time of freezing procedures and eliminating the need for programmable cell freezers required for conventional slow freezing. Moreover, equilibrium freezing may not be the most advantageous method to cryopreserve oocytes as they are damaged due to long exposure to temperatures near $0^{\circ} \mathrm{C}[20]$. Vitrification is the alternative method of cryopreservation which uses an ultra rapid cooling rate, eliminating the need for programmable freezing equipment. Furthermore, the vitrification technique uses high concentrations of cryoprotectants which avoids water precipitation, preventing intracellular ice crystal formation [3]. In the last several years, almost all advancements in oocyte cryopreservation have been made using vitrification techniques, and their use for oocyte and embryo cryopreservation will undoubtedly increase in the future [26].

In 1985, vitrification of mouse embryos emerged as an alternative approach to traditional slow freezing methods [71]. However, the first successful mouse embryo vitrification was documented in 1993 [72]. Bovine oocytes are able to develop to the blastocyst stage following high cooling rates [65], and pregnancies have been achieved following vitrification of human oocytes [73, 74].

Many variables in the vitrification process exist that can profoundly influence the survival rate of oocytes. The extent of injury and the differences in survival and developmental rates are variable depending on the species, developmental stage, and origin [3]. The type and concentration of the cryoprotectant, the temperature of the vitrification solution at the time of cell exposure, and the duration of exposure to the final cryoprotectant before plunging in $\mathrm{LN}_{2}$ are important factors to improve the survival rates. The exposure time of oocytes and embryos to cryoprotectants may be shortened, or they are often pre-equilibrated in a vitrification solution containing a lower concentration of permeating cryoprotectants to avoid any anticipated toxic shock resulting from exposure to higher concentrations of cryoprotectants in a final vitrification solution [75-77]. However, it is not yet clear whether a pre-exposure to lower concentrations of vitrification is necessary and, if so, what is the optimal time for such exposure. During warming, the main biophysical factor causing cellular disruption is osmotic injury, which can occur during the removal of penetrating cryoprotectants from the cell. The ideal time for oocytes to be suspended in warming medium is still uncertain. If the time is insufficient, cryoprotectants may not be completely removed; however if left too long, osmotic swelling can occur, especially given that cryopreserved cells are much more sensitive than nonfrozen cells $[78]$.

Moreover, the type of cryodevice used for vitrification influences the cooling rate and size of the vapor coat. Oocytes from humans [79-81], pigs [82], horses [83], sheep [58, 84], cattle [85], and buffalo $[86,87]$ have developed in vitro following cryopreservation using cryotop. Although results are inconsistent, meiotic stage is also thought to contribute to oocyte survivability following cryopreservation [32, 8892]. The ideal strategy to improve the success of vitrification includes increasing the speed of thermal conduction and decreasing the concentration of cryoprotectants [53].

5.1. Bovine. Although the cryopreservation of bovine oocytes remains a challenge, some of the most encouraging results among domestic animals have been obtained in the bovine, where offspring have been born from immature and mature vitrified oocytes following IVF and culture $[93,94]$. Vajta [82] reported a $25 \%$ blastocyst rate on day 8 following vitrification using open-pulled straw, thawing, IVF, and culture in vitro. Bovine oocytes are much more 
cryostable than porcine oocytes due to less lipid contents and intracellular lipid droplets and vesicles, thus porcine oocytes are much more difficult to successfully cryopreserve than bovine [95]. GV stage bovine oocytes have homogenous lipid droplets that show little change following cooling; however their large size and low surface: volume ratio makes it difficult for water and cryoprotectants to move across the plasma membrane [96].

In order to obtain higher blastocyst formation and healthy offspring following vitrification, good quality bovine oocytes should be used. Oocytes matured in vivo demonstrate a significantly higher maturation rate and blastocyst formation than those matured in vitro $[97,98]$. Oocytes obtained from large growing follicles that are not in the presence of a dominant follicle and have large enough diameters to be considered competent also assist in improving cryopreservation rates of bovine oocytes [20]. Moreover, a partial removal of cumulus cells several hours after the onset of IVM may facilitate cryoprotectant penetration while still maintaining supportive and nutritive roles [20].

Although modifying cryopreservation methods to fit the cell type being cryopreserved may be more preferable, modifying cells to fit the cryopreservation procedure has also been proposed to improve survival rates [96]. The cleavage rate and 8-cell embryo stage improved significantly after treating bovine oocytes with cholesterol-loaded cyclodextrin [99]. The centrifugation of mature bovine oocytes for partial removal of cytoplasmic lipid droplets prior to vitrification reduced the incidence of polyspermy [100]. Modifying the lipid content, removing serum albumin from media, and the addition of a membrane stabilizer such as trehalose to the cytoplasm are worth investigating factors [96].

5.2. Other Farm Animals. Although offspring have been produced after the transfer of embryos from frozen-thawed oocytes in several species $[45,101]$, the overall success rate has been low, primarily due to the decreased rate of fertilization following freezing and thawing [54]. Similar to embryos, porcine oocytes are highly sensitive to low temperatures. Consequently there is yet to be viable piglets born from cryopreserved oocytes in spite of blastocyst development following cryopreservation of MII stage oocytes $[36,82]$. The meiotic spindle of porcine oocytes is extremely sensitive to cryopreservation, resulting in impaired development at meiosis-II [21]. However, the main cause of poor survival following vitrification can be attributed to the high intracellular lipid content in porcine oocytes. Porcine GV oocytes contain 2.4-fold more lipid droplets than bovine oocytes [102]. Additionally, dark homogenous lipid droplets as well as grey ones with electron-lucent streaks change morphologically from round to spherical with lucent streaks during cooling $[3,95]$. However, the removal of cytoplasmic lipid droplets using delipidation increases their freezing tolerance [103]. A recent study combined the removal of cytoplasmic lipid droplets with microtubule stabilization and found that vitrified porcine IVM MII stage oocytes could develop to the blastocyst stage and maintain the ability to develop into fetuses [36]. Furthermore, the use of cholesterol-loaded cyclodextrin to increase the cholesterol content of oocyte membranes has improved cryotolerance [96].

In equine, immature oocytes undergo significant damage during controlled freezing. Less than $16 \%$ reach MII stage during post thaw maturation, which is less than the $50-80 \%$ of non-cryopreserved oocytes [104, 105]. Although MII rates are higher in equine oocytes that have been vitrified (28-46\%), approximately $50 \%$ of oocytes reaching MII stage exhibit spindle abnormalities and poor developmental competence $[28,106,107]$. Much of the damage to equine oocytes is on the mitochondria and gap junctions between the oocyte and surrounding cumulus-corona radiata cells and these interactions are critical for successful maturation and developmental competence [34].

Currently, a limited number of studies have been done on the vitrification of small ruminant oocytes, especially in sheep, where poor developmental rates are obtained following immature [108, 109] and mature [58] oocyte vitrification. Poor ovine oocyte cryopreservation has been attributed to the damage to enzymes such as mitogenactivated protein (MAPK) kinase, critical for oocyte maturation and subsequent embryo development. The denudation of immature ovine oocytes prior to vitrification increased survival and maturation ability, however, the process also decreases maturation promoting factor (MPF) and MAP kinase levels, which influence meiotic and mitotic cell cycle regulation and developmental competence [31]. Current investigations are focusing on the factors influencing the ability of vitrified ovine oocytes to undergo IVF and develop further.

Despite the recent advancements, the cryopreservation of oocytes of most mammalian species remains a challenge due to their complex structure. Although there has been increasing number of publications regarding vitrification of oocytes, more research is required to further elucidate the species-specific mechanisms influencing poor survivability following vitrification. Moreover, additional examination of modifying oocytes to fit the cryopreservation technique may be necessary, especially in species whose oocytes have extremely high lipid contents.

\section{Conclusions}

The loss of farm animal genetic resources is occurring at alarming rates across the globe. Traditions, cultural values, and safeguarding diversity for an unpredictable future are all driving forces for genetic conservation, which is a global responsibility. Preserving live animals permits further evolution of breeds; however, in many cases, in situ conservation strategies are not practical or adequate. Therefore, establishing genetic resource banks to conserve the current genetic status would provide a crucial interface between ex situ and in situ conservation strategies.

Cryopreservation of oocytes is a crucial step for the conservation of the female portion of genetics; however despite decades of research, it remains a challenge in virtually all species due to the complex structure of the oocyte. Conventional slow freezing commonly leads to intracellular ice crystallization and cell damage, and although vitrification 
of bovine oocytes is gaining popularity, it is still challenging due to oocyte's complex structure and sensitivity to chilling. Vitrification is a relatively simple and inexpensive method of cryopreserving oocytes; however, despite the tireless efforts over the past 20 years, vitrification has yet to achieve convincing results capable of widespread application. It is critical that researchers achieve more consistent results and establish a "universal" protocol that can be applied for the cryopreservation of oocytes at different developmental stages.

\section{References}

[1] W. V. Holt and A. R. Pickard, "Role of reproductive technologies and genetic resource banks in animal conservation," Reviews of Reproduction, vol. 4, no. 3, pp. 143-150, 1999.

[2] J. Hanks, "Conservation strategies for Africa's large mammals," Reproduction, Fertility and Development, vol. 13, no. 7-8, pp. 459-468, 2001.

[3] R. M. Pereira and C. C. Marques, "Animal oocyte and embryo cryopreservation," Cell and Tissue Banking, vol. 9, no. 4, pp. 267-277, 2008.

[4] S. Ledda, G. Leoni, L. Bogliolo, and S. Naitana, "Oocyte cryopreservation and ovarian tissue banking," Theriogenology, vol. 55, no. 6, pp. 1359-1371, 2001.

[5] C. M. Checura and G. E. Seidel Jr., "Effect of macromolecules in solutions for vitrification of mature bovine oocytes," Theriogenology, vol. 67, no. 5, pp. 919-930, 2007.

[6] D. L. Patterson and F. G. Silversides, "Farm Animal Genetic Resource Conservation. Why and how?” 2003, http://www .cfagrf.com/Farm_Animal_Gentetic_Resource_Conservation_ Why_and_How.htm.

[7] T. Buerkle, "FAO sounds alarm on loss of livestock breeds," Food and Agriculture Organization of the United Nations, 2007, http://www.fao.org/.

[8] S. M. H. Andrabi and W. M. C. Maxwell, "A review on reproductive biotechnologies for conservation of endangered mammalian species," Animal Reproduction Science, vol. 99, no. 3-4, pp. 223-243, 2007.

[9] L. Skuterud, E. Gaare, I. M. Eikelmann, K. Hove, and E. Steinnes, "Chernobyl radioactivity persists in reindeer," Journal of Environmental Radioactivity, vol. 83, no. 2, pp. 231-252, 2005.

[10] T. Sazykina and I. I. Kryshev, "Radiation effects in wild terrestrial vertebrates-the EPIC collection," Journal of Environmental Radioactivity, vol. 88, no. 1, pp. 11-48, 2006.

[11] A. K. Mishra, A. L. Arora, S. Kumar, and L. L. L. Prince, "Studies on effect of Booroola (FecB) genotype on lifetime ewes' productivity efficiency, litter size and number of weaned lambs in Garole $\times$ Malpura sheep," Animal Reproduction Science, vol. 113, no. 1-4, pp. 293-298, 2009.

[12] P. J. Boettcher, A. Stella, F. Pizzi, and G. Gandini, “The combined use of embryos and semen for cryogenic conservation of mammalian livestock genetic resources," Genetics Selection Evolution, vol. 37, no. 6, pp. 657-675, 2005.

[13] B. Demirci, J. Lornage, B. Salle, M. T. Poirel, J. F. Guerin, and M. Franck, "The cryopreservation of ovarian tissue: uses and indications in veterinary medicine," Theriogenology, vol. 60, no. 6, pp. 999-1010, 2003.

[14] FAO, "The state of the world's animal genetic resources for food and agriculture," Commission on Genetic Resources for Food and Agriculture; Food and Agriculture Organization of the United Nations, Rome, Italy, 2007.
[15] L. A. Johnston and R. C. Lacy, "Genome resource banking for species conservation: selection of sperm donors," Cryobiology, vol. 32, no. 1, pp. 68-77, 1995.

[16] E. J. Woods, J. D. Benson, Y. Agca, and J. K. Critser, "Fundamental cryobiology of reproductive cells and tissues," Cryobiology, vol. 48, no. 2, pp. 146-156, 2004.

[17] H. Niemann and D. Rath, "Progress in reproductive biotechnology in swine," Theriogenology, vol. 56, no. 8, pp. 12911304, 2001.

[18] S. Hochi, T. Fujimoto, and N. Oguri, "Large equine blastocysts are damaged by vitrification procedures," Reproduction, Fertility and Development, vol. 7, no. 1, pp. 113-117, 1995.

[19] T. Fair, P. Lonergan, A. Dinnyes et al., "Ultrastructure of bovine blastocysts following cryopreservation: effect of method of blastocyst production," Molecular Reproduction and Development, vol. 58, no. 2, pp. 186-195, 2001.

[20] A. Massip and I. Donnay, "Cryopreservation of bovine oocytes: current status and recent developments," Reproduction Nutrition Development, vol. 43, no. 4, pp. 325-330, 2003.

[21] S. U. Chen, Y. R. Lien, K. H. Chao, H. N. Ho, Y. S. Yang, and T. Y. Lee, "Effects of cryopreservation on meiotic spindles of oocytes and its dynamics after thawing: clinical implications in oocyte freezing-a review article," Molecular and Cellular Endocrinology, vol. 202, no. 1-2, pp. 101-107, 2003.

[22] T. Otoi, K. Yamamoto, N. Koyama, S. Tachikawa, and T. Suzuki, "A frozen-thawed in vitro-matured bovine oocyte derived calf with normal growth and fertility," Journal of Veterinary Medical Science, vol. 58, no. 8, pp. 811-813, 1996.

[23] L. J. Maclellan, E. M. Carnevale, M. A. Coutinho da Silva, C. F. Scoggin, J. E. Bruemmer, and E. L. Squires, "Pregnancies from vitrified equine oocytes collected from superstimulated and non-stimulated mares," Theriogenology, vol. 58, no. 5, pp. 911-919, 2002.

[24] P. Mazur, "Cryobiology: the freezing of biological systems," Science, vol. 168, no. 3934, pp. 939-949, 1970.

[25] J. Wolfe and G. Bryant, "Cellular cryobiology: thermodynamic amd mechanical effects," International Journal of Refrigeration, vol. 24, no. 5, pp. 438-450, 2001.

[26] G. Vajta and M. Kuwayama, "Improving cryopreservation systems," Theriogenology, vol. 65, no. 1, pp. 236-244, 2006.

[27] D. A. Gook, S. M. Osborn, and W. I. H. Johnston, "Cryopreservation of mouse and human oocytes using 1,2propanediol and the configuration of the meiotic spindle," Human Reproduction, vol. 8, no. 7, pp. 1101-1109, 1993.

[28] T. Tharasanit, S. Colleoni, C. Galli, B. Colenbrandera, and T. A. E. Stout, "Protective effects of the cumulus-corona radiata complex during vitrification of horse oocytes," Reproduction, vol. 137, no. 3, pp. 391-401, 2009.

[29] A. Martino, J. W. Pollard, and S. P. Leibo, "Effect of chilling bovine oocytes on their developmental competence," Molecular Reproduction and Development, vol. 45, no. 4, pp. 503-512, 1996.

[30] G. Vajta, "Vitrification of the oocytes and embryos of domestic animals," Animal Reproduction Science, vol. 60-61, pp. 357-364, 2000.

[31] L. Bogliolo, F. Ariu, S. Fois et al., "Morphological and biochemical analysis of immature ovine oocytes vitrified with or without cumulus cells," Theriogenology, vol. 68, no. 8, pp. 1138-1149, 2007.

[32] H. Men, R. L. Monson, and J. J. Rutledge, "Effect of meiotic stages and maturation protocols on bovine oocyte's resistance to cryopreservation," Theriogenology, vol. 57, no. 3, pp. 1095-1103, 2002. 
[33] Y. Agca, J. Liu, J. J. Rutledge, E. S. Critser, and J. K. Critser, "Effect of osmotic stress on the developmental competence of germinal vesicle and metaphase II stage bovine cumulus oocyte complexes and its relevance to cryopreservation," Molecular Reproduction and Development, vol. 55, no. 2, pp. 212-219, 2000.

[34] S. Hochi, M. Kozawa, T. Fujimoto, E. Hondo, J. Yamada, and N. Oguri, "In vitro maturation and transmission electron microscopic observation of horse oocytes after vitrification," Cryobiology, vol. 33, no. 3, pp. 300-310, 1996.

[35] C. M. H. Combelles, N. A. Cekleniak, C. Racowsky, and D. F. Albertini, "Assessment of nuclear and cytoplasmic maturation in in-vitro matured human oocytes," Human Reproduction, vol. 17, no. 4, pp. 1006-1016, 2002.

[36] B. Ogawa, S. Ueno, N. Nakayama et al., "Developmental ability of porcine in vitro matured oocytes at the meiosis II stage after vitrification," Journal of Reproduction and Development, vol. 56, no. 3, pp. 356-361, 2010.

[37] G. Schatten, C. Simerly, and H. Schatten, "Microtubule configurations during fertilization, mitosis, and early development in the mouse and the requirement for egg microtubulemediated motility during mammalian fertilization," Proceedings of the National Academy of Sciences of the United States of America, vol. 82, no. 12, pp. 4152-4156, 1985.

[38] A. Eroglu, T. L. Toth, and M. Toner, "Alterations of the cytoskeleton and polyploidy reduced by cryopreservation of metaphase II mouse oocytes," Fertility and Sterility, vol. 69, no. 5, pp. 944-957, 1998.

[39] R. Morató, D. Izquierdo, J. L. Albarracín et al., "Effects of pre-treating in vitro-matured bovine oocytes with the cytoskeleton stabilizing agent taxol prior to vitrification," Molecular Reproduction and Development, vol. 75, no. 1, pp. 191-201, 2008.

[40] S. Succu, G. G. Leoni, F. Berlinguer et al., "Effect of vitrification solutions and cooling upon in vitro matured prepubertal ovine oocytes," Theriogenology, vol. 68, no. 1, pp. 107-114, 2007.

[41] A. Mavrides and D. Morroll, "Cryopreservation of bovine oocytes: is cryoloop vitrification the future to preserving the female gamete?" Reproduction Nutrition Development, vol. 42, no. 1, pp. 73-80, 2002.

[42] C. Rojas, M. J. Palomo, J. L. Albarracín, and T. Mogas, "Vitrification of immature and in vitro matured pig oocytes: study of distribution of chromosomes, microtubules, and actin microfilaments," Cryobiology, vol. 49, no. 3, pp. 211220, 2004.

[43] R. Kazem, L. A. Thompson, A. Srikantharajah, M. A. Laing, M. P. R. Hamilton, and A. Templeton, "Cryopreservation of human oocytes and fertilization by two techniques: in-vitro fertilization and intracytoplasmic sperm injection," Human Reproduction, vol. 10, no. 10, pp. 2650-2654, 1995.

[44] N. Cocchia, F. Ciani, M. Russo et al., "Immature cat oocyte vitrification in open pulled straws (OPSs) using a cryoprotectant mixture," Cryobiology, vol. 60, no. 2, pp. 229234, 2010.

[45] C. Chen, "Pregnancy after human oocyte cryopreservation," The Lancet, vol. 1, no. 8486, pp. 884-886, 1986.

[46] S. J. Paynter, B. J. Fuller, and R. W. Shaw, "Temperature dependence of mature mouse oocyte membrane permeabilities in the presence of cryoprotectant," Cryobiology, vol. 34, no. 2, pp. 122-130, 1997.

[47] C.-C. Chang, L.-Y. Sung, T. Amano, X. C. Tian, X. Yang, and Z. P. Nagy, "Nuclear transfer and oocyte cryopreservation,"
Reproduction, Fertility and Development, vol. 21, no. 1, pp. 37-44, 2009.

[48] A. M. van Wagtendonk-de Leeuw, J. H. G. den Daas, and W. F. Rall, "Field trial to compare pregnancy rates of bovine embryo cryopreservation methods: vitrification and onestep dilution versus slow freezing and three-step dilution," Theriogenology, vol. 48, no. 7, pp. 1071-1084, 1997.

[49] Y. Agca, R. L. Monson, D. L. Northey, O. A. Mazni, D. M. Schaefer, and J. J. Rutledge, "Transfer of fresh and cryopreserved ivp bovine embryos: normal calving, birth weight and gestation lengths," Theriogenology, vol. 50, no. 1, pp. 147-162, 1998.

[50] G. Vajta, P. Holm, M. Kuwayama et al., "Open pulled straw (OPS) vitrification: a new way to reduce cryoinjuries of bovine ova and embryos," Molecular Reproduction and Development, vol. 51, no. 1, pp. 53-58, 1998.

[51] F. Berthelot, F. Martinat-Botté, A. Locatelli, C. Perreau, and M. Terqui, "Piglets born after vitrification of embryos using the open pulled straw method," Cryobiology, vol. 41, no. 2, pp. 116-124, 2000.

[52] G. Vajta and Z. P. Nagy, "Are programmable freezers still needed in the embryo laboratory? Review on vitrification," Reproductive BioMedicine Online, vol. 12, no. 6, pp. 779-796, 2006.

[53] Y. Orief, A. Schultze-Mosgau, K. Dafopoulos, and S. AlHasani, "Vitrification: will it replace the conventional gamete cryopreservation techniques?" Middle East Fertility Society Journal, vol. 10, no. 3, pp. 171-184, 2005.

[54] A. T. Palasz and R. J. Mapletoft, "Cryopreservation of mammalian embryos and oocytes: recent advances," Biotechnology Advances, vol. 14, no. 2, pp. 127-149, 1996.

[55] L. L. Kuleshova, D. R. MacFarlane, A. O. Trounson, and J. M. Shaw, "Sugars exert a major influence on the vitrification properties of ethylene glycol-based solutions and have low toxicity to embryos and oocytes," Cryobiology, vol. 38, no. 2, pp. 119-130, 1999.

[56] S.-U. Chen, Y.-R. Lien, H.-F. Chen, K.-H. Chao, N.-N. Ho, and Y.-S. Yang, "Open pulled straws for vitrification of mature mouse oocytes preserve patterns of meiotic spindles and chromosomes better than conventional straws," Human Reproduction, vol. 15, no. 12, pp. 2598-2603, 2000.

[57] F. Le Gal and A. Massip, "Cryopreservation of cattle oocytes: effects of meiotic stage, cycloheximide treatment, and vitrification procedure," Cryobiology, vol. 38, no. 4, pp. 290-300, 1999.

[58] S. Succu, G. G. Leoni, D. Bebbere et al., "Vitrification devices affect structural and molecular status of in vitro matured ovine oocytes," Molecular Reproduction and Development, vol. 74, no. 10, pp. 1337-1344, 2007.

[59] R. Morató, D. Izquierdo, M. T. Paramio, and T. Mogas, "Embryo development and structural analysis of in vitro matured bovine oocytes vitrified in flexipet denuding pipettes," Theriogenology, vol. 70, no. 9, pp. 1536-1543, 2008.

[60] S. W. Hong, H. M. Chung, J. M. Lim, J. J. Ko, T. K. Yoon, and K. Y. Cha, "Improved human oocyte development after vitrification: a comparison of thawing methods," Fertility and Sterility, vol. 72, no. 1, pp. 142-146, 1999.

[61] J. Liebermann and M. J. Tucker, "Effect of carrier system on the yield of human oocytes and embryos as assessed by survival and developmental potential after vitrification," Reproduction, vol. 124, no. 4, pp. 483-489, 2002.

[62] T. Kurokawa, T. Kinoshita, T. Ito, H. Saito, and T. Hotta, "Detection of minimal residual disease in B cell lymphoma 
by a PCR-mediated RNase protection assay," Leukemia, vol. 10, no. 7, pp. 1222-1231, 1996.

[63] Y. Abe, K. Hara, H. Matsumoto et al., "Feasibility of a nylonmesh holder for vitrification of bovine germinal vesicle oocytes in subsequent production of viable blastocysts," Biology of Reproduction, vol. 72, no. 6, pp. 1416-1420, 2005.

[64] R. Morató, D. Izquierdo, M. T. Paramio, and T. Mogas, "Cryotops versus open-pulled straws (OPS) as carriers for the cryopreservation of bovine oocytes: effects on spindle and chromosome configuration and embryo development," Cryobiology, vol. 57, no. 2, pp. 137-141, 2008.

[65] A. Martino, N. Songsasen, and S. P. Leibo, "Development into blastocysts of bovine oocytes cryopreserved by ultra-rapid cooling," Biology of Reproduction, vol. 54, no. 5, pp. 10591069, 1996.

[66] S. Ledda, L. Bogliolo, S. Succu et al., "Oocyte cryopreservation: oocyte assessment and strategies for improving survival," Reproduction, Fertility and Development, vol. 19, no. 1, pp. 13-23, 2007.

[67] K. Kumamoto, H. Wang, H. Yamashiro, and T. Terada, "Easy and rapid method for the determination of gene expression in cumulus cells incubated for oocyte maturation," Reproductive Biology and Endocrinology, vol. 3, Article ID 59, 2005.

[68] S. Hamano, A. Koikeda, M. Kuwayama, and T. Nagai, "Fullterm development of in vitro-matured, vitrified and fertilized bovine oocytes," Theriogenology, vol. 38, no. 6, pp. 10851090, 1992.

[69] E. Porcu, R. Fabbri, R. Seracchioli, P. M. Ciotti, O. Magrini, and C. Flamigni, "Birth of a healthy female after intracytoplasmic sperm injection of cryopreserved human oocytes," Fertility and Sterility, vol. 68, no. 4, pp. 724-726, 1997.

[70] A. D. Vieira, F. Forell, C. Feltrin, and J. L. Rodrigues, "Calves born after direct transfer of vitrified bovine in vitro-produced blastocysts derived from vitrified immature oocytes," Reproduction in Domestic Animals, vol. 43, no. 3, pp. 314-318, 2008.

[71] W. F. Rall and G. M. Fahy, "Ice-free cryopreservation of mouse embryos at $-196^{\circ} \mathrm{C}$ by vitrification," Nature, vol. 313, no. 6003 , pp. 573-575, 1985.

[72] J. Ali and J. N. Shelton, "Vitrification of preimplantation stages of mouse embryos," Journal of Reproduction and Fertility, vol. 98, no. 2, pp. 459-465, 1993.

[73] T. K. Yoon, H. M. Chung, J. M. Lim, S. Y. Han, J. J. Ko, and K. Y. Cha, "Pregnancy and delivery of healthy infants developed from vitrified oocytes in a stimulated in vitro fertilizationembryo transfer program," Fertility and Sterility, vol. 74, no. 1, pp. 180-181, 2000.

[74] L. Kuleshova, L. Gianaroli, C. Magli, A. Ferraretti, and A. Trounson, "Birth following vitrification of a small number of human oocytes," Human Reproduction, vol. 14, no. 12, pp. 3077-3079, 1999.

[75] T. Kono, O. Y. Kwon, and T. Nakahara, "Development of vitrified mouse oocytes after in vitro fertilization," Cryobiology, vol. 28 , no. 1 , pp. 50-54, 1991.

[76] M. J. Wood, C. Barros, C. J. Candy, J. Carroll, J. Melendez, and D. G. Whittingham, "High rates of survival and fertilization of mouse and hamster oocytes after vitrification in dimethylsulphoxide," Biology of Reproduction, vol. 49, no. 3, pp. 489-495, 1993.

[77] A. Dhali, R. S. Manik, S. K. Das, S. K. Singla, and P. Palta, "Post-vitrification survival and in vitro maturation rate of buffalo (Bubalus bubalis) oocytes: effect of ethylene glycol concentration and exposure time," Animal Reproduction Science, vol. 63, no. 3-4, pp. 159-165, 2000.
[78] P. B. Pedro, S. E. Zhu, N. Makino, T. Sakurai, K. Edashige, and M. Kasai, "Effects of hypotonic stress on the survival of mouse oocytes and embryos at various stages," Cryobiology, vol. 35, no. 2, pp. 150-158, 1997.

[79] E. Lucena, D. P. Bernal, C. Lucena, A. Rojas, A. Moran, and A. Lucena, "Successful ongoing pregnancies after vitrification of oocytes," Fertility and Sterility, vol. 85, no. 1, pp. 108-111, 2006.

[80] M. Antinori, E. Licata, G. Dani, F. Cerusico, C. Versaci, and S. Antinori, "Cryotop vitrification of human oocytes results in high survival rate and healthy deliveries," Reproductive BioMedicine Online, vol. 14, no. 1, pp. 73-79, 2007.

[81] M. Kuwayama, "Highly efficient vitrification for cryopreservation of human oocytes and embryos: the Cryotop method," Theriogenology, vol. 67, no. 1, pp. 73-80, 2007.

[82] Y. Liu, Y. Du, L. Lin et al., "Comparison of efficiency of open pulled straw (OPS) and cryotop vitrification for cryopreservation of in vitro matured pig oocytes," CryoLetters, vol. 29, no. 4, pp. 315-320, 2008.

[83] L. Bogliolo, F. Ariu, I. Rosati et al., "Vitrification of immature and in vitro matured horse oocytes," Reproduction, Fertility and Development, vol. 18, pp. 149-150, 2006.

[84] B. Ebrahimi, M. R. Valojerdi, P. Eftekhari-Yazdi, and H. Baharvand, "In vitro maturation, apoptotic gene expression and incidence of numerical chromosomal abnormalities following cryotop vitrification of sheep cumulus-oocyte complexes," Journal of Assisted Reproduction and Genetics, vol. 27, no. 5, pp. 239-246, 2010.

[85] N. Sripunya, T. Somfai, Y. Inaba, T. Nagai, K. Imai, and R. Parnpai, "A comparison of cryotop and solid surface vitrification methods for the cryopreservation of in vitro matured bovine oocytes," Journal of Reproduction and Development, vol. 56, no. 1, pp. 176-181, 2010.

[86] B. Gasparrini, L. Attanasio, A. De Rosa, E. Monaco, R. Di Palo, and G. Campanile, "Cryopreservation of in vitro matured buffalo (Bubalus bubalis) oocytes by minimum volumes vitrification methods," Animal Reproduction Science, vol. 98, no. 3-4, pp. 335-342, 2007.

[87] L. Attanasio, L. Boccia, G. Vajta et al., "Cryotop vitrification of buffalo (Bubalus Bubalis) in vitro matured oocytes: effects of cryoprotectant concentrations and warming procedures," Reproduction in Domestic Animals. In press.

[88] S. Hochi, K. Ito, M. Hirabayashi et al., "Effect of nuclear stages during IVM on the survival of vitrified warmed bovine oocytes," Theriogenology, vol. 49, pp. 787-796, 1998.

[89] A. E. Hurtt, F. Landim-Alvarenga, G. E. Scidel Jr., and E. L. Squires, "Vitrification of immature and mature equine and bovine oocytes in an ethylene glycol, ficoll and sucrose solution using open-pulled straws," Theriogenology, vol. 54, no. 1, pp. 119-128, 2000.

[90] J. L. Albarracín, R. Morató, D. Izquierdo, and T. Mogas, "Vitrification of calf oocytes: effects of maturation stage and prematuration treatment on the nuclear and cytoskeletal components of oocytes and their subsequent development," Molecular Reproduction and Development, vol. 72, no. 2, pp. 239-249, 2005.

[91] G. T. Sharma and K. Loganathasamy, "Effect of meiotic stages during in vitro maturation on the survival of vitrifiedwarmed buffalo oocytes," Veterinary Research Communications, vol. 31, no. 7, pp. 881-893, 2007.

[92] V. Magnusson, W. B. Feitosa, M. D. Goissis et al., "Bovine oocyte vitrification: effect of ethylene glycol concentrations and meiotic stages," Animal Reproduction Science, vol. 106, no. 3-4, pp. 265-273, 2008. 
[93] A. D. Vieira, A. Mezzalira, D. P. Barbieri, R. C. Lehmkuhl, M. I. B. Rubin, and G. Vajta, "Calves born after open pulled straw vitrification of immature bovine oocytes," Cryobiology, vol. 45, no. 1, pp. 91-94, 2002.

[94] K. Papis, M. Shimizu, and Y. Izaike, "Factors affecting the survivability of bovine oocytes vitrified in droplets," Theriogenology, vol. 54, no. 5, pp. 651-658, 2000.

[95] V. Isachenko, E. Isachenko, H. W. Michelmann et al., "Lipolysis and ultrastructural changes of intracellular lipid vesicles after cooling of bovine and porcine GV-oocytes," Anatomia, Histologia, Embryologia, vol. 30, no. 6, pp. 333$338,2001$.

[96] G. E. Seidel Jr., "Modifying oocytes and embryos to improve their cryopreservation," Theriogenology, vol. 65, no. 1, pp. 228-235, 2006.

[97] A. Van Soom and A. de Kruif, "A comparative study of in vivo and in vitro derived bovine embryos," in Proceedings of the 12th International Congress on Animal Reproduction, vol. 3, pp. 1365-1367, 1992.

[98] D. Rizos, F. Ward, P. Duffy, M. P. Boland, and P. Lonergan, "Consequences of bovine oocyte maturation, fertilization or early embryo development in vitro versus in vivo: implications for blastocyst yield and blastocyst quality," Molecular Reproduction and Development, vol. 61, no. 2, pp. 234-248, 2002.

[99] G. Horvath and G. E. Seidel Jr., "Vitrification of bovine oocytes after treatment with cholesterol-loaded methyl- $\beta$ cyclodextrin," Theriogenology, vol. 66, no. 4, pp. 1026-1033, 2006.

[100] T. Otoi, K. Yamamoto, N. Koyama et al., "Cryopreservation of mature bovine oocytes following centrifugation treatment," Cryobiology, vol. 34, no. 1, pp. 36-41, 1997.

[101] E. Fuku, T. Kojima, Y. Shioya, G. J. Marcus, and B. R. Downey, "In vitro fertilization and development of frozen-thawed bovine oocytes," Cryobiology, vol. 29, no. 4, pp. 485-492, 1992.

[102] G. Genicot, J. L. M. R. Leroy, A. Van Soom, and I. Donnay, "The use of a fluorescent dye, Nile red, to evaluate the lipid content of single mammalian oocytes," Theriogenology, vol. 63, no. 4, pp. 1181-1194, 2005.

[103] H. Nagashima, R. D. A. Cameron, M. Kuwayama et al., "Survival of porcine delipated oocytes and embryos after cryopreservation by cytoskeleton stabilizer," Animal Reproduction Science, vol. 110, pp. 46-55, 2009.

[104] S. Hochi, T. Fujimoto, Y. H. Choi, J. Braun, and N. Oguri, "Cryopreservation of equine oocytes by 2-step freezing," Theriogenology, vol. 42, no. 7, pp. 1085-1094, 1994.

[105] T. Tharasanit, B. Colenbrander, and T. A. E. Stout, "Effect of maturation stage at cryopreservation on post-thaw cytoskeleton quality and fertilizability of equine oocytes," Molecular Reproduction and Development, vol. 73, no. 5, pp. 627-637, 2006.

[106] T. Tharasanit, S. Colleoni, G. Lazzari, B. Colenbrander, C. Galli, and T. A. E. Stout, "Effect of cumulus morphology and maturation stage on the cryopreservability of equine oocytes," Reproduction, vol. 132, no. 5, pp. 759-769, 2006.

[107] A. E. Hurtt, F. Landim-Alvarenga, G. E. Scidel Jr., and E. L. Squires, "Vitrification of immature and mature equine and bovine oocytes in an ethylene glycol, ficoll and sucrose solution using open-pulled straws," Theriogenology, vol. 54, no. 1, pp. 119-128, 2000.

[108] V. Isachenko, J. L. Alabart, F. Nawroth, E. Isachenko, G. Vajta, and J. Folch, "The open pulled straw vitrification of ovine
GV-oocytes: positive effect of rapid cooling or rapid thawing or both?" Cryo-Letters, vol. 22, no. 3, pp. 157-162, 2001.

[109] A. M. Al-aghbari and A. R. Menino Jr., "Survival of oocytes recovered from vitrified sheep ovarian tissues," Animal Reproduction Science, vol. 71, no. 1-2, pp. 101-110, 2002. 

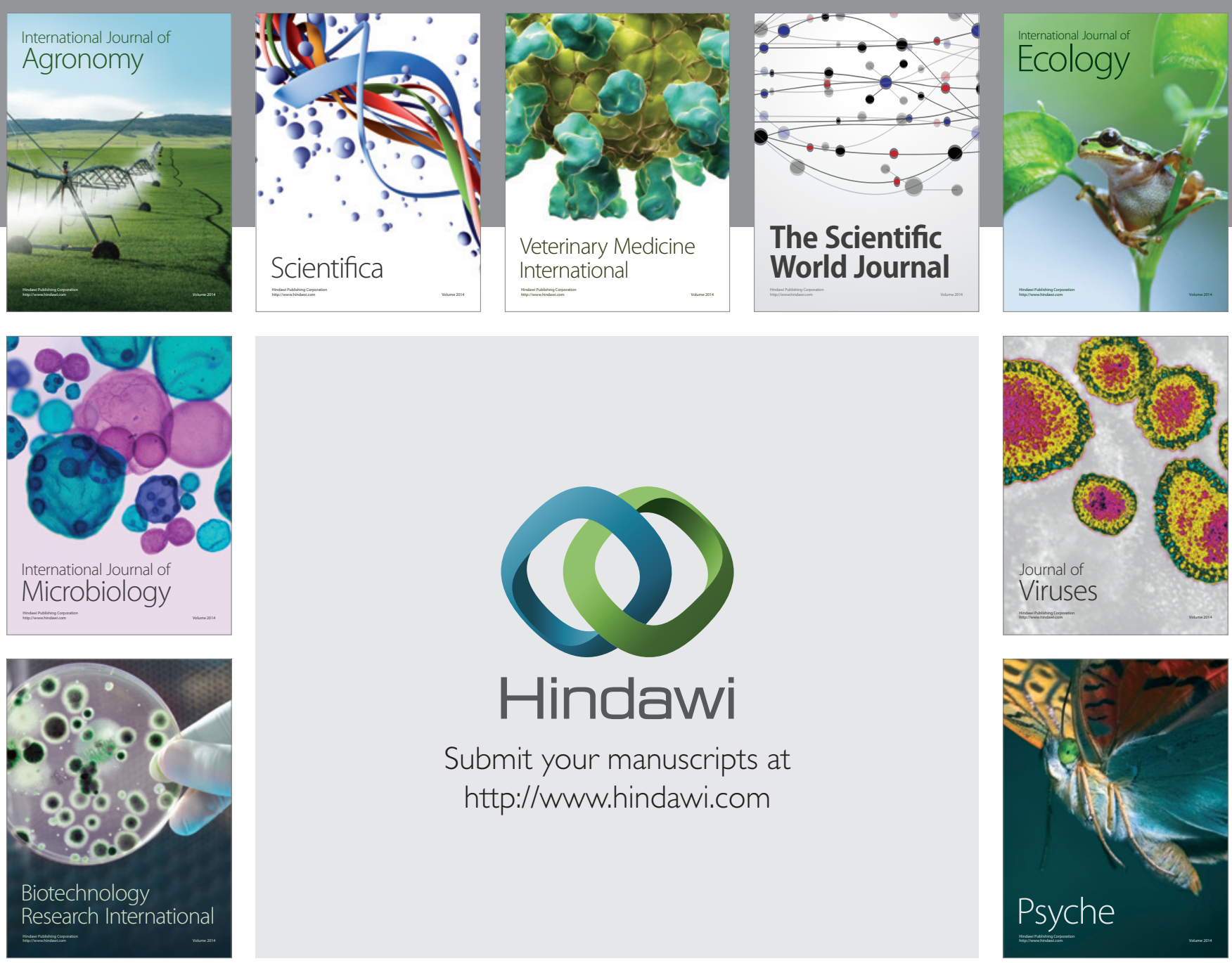

Submit your manuscripts at

http://www.hindawi.com
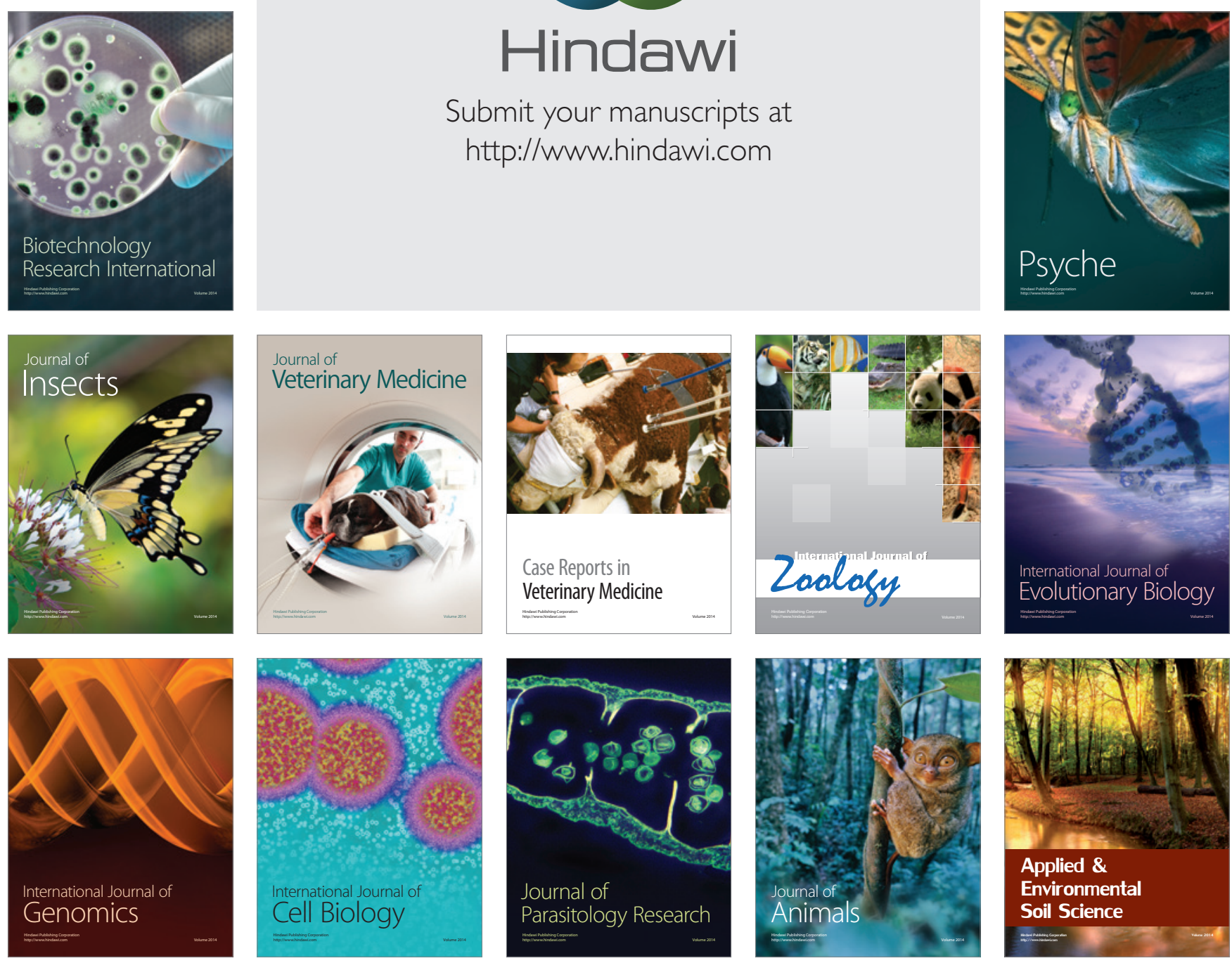